\title{
Effect of Annexin A1 gene on the proliferation and invasion of esophageal squamous cell carcinoma cells and its regulatory mechanisms
}

\author{
GAOHUA HAN ${ }^{1}$, KAIJIN LU $^{2}$, JUNXING HUANG $^{1}$, JUN YE $^{3}$, SHENGBIN DAI $^{1}$, YUNYAO YE $^{1}$ and LIXIN ZHANG ${ }^{3}$ \\ Departments of ${ }^{1}$ Oncology, ${ }^{2}$ Chest Surgery and ${ }^{3}$ Central Laboratory, Taizhou People's Hospital Affiliated to \\ Nantong University, Taizhou, Jiangsu 225300, P.R. China
}

Received January 26, 2016; Accepted December 5, 2016

DOI: $10.3892 / \mathrm{ijmm} .2016 .2840$

\begin{abstract}
The aim of this study was to examine the effect of Annexin A1 (ANXA1) on the proliferation, migration and invasion of esophageal squamous cell carcinoma (ESCC) cells and its possible mechanisms of action. After constructing the ANXA1 overexpression plasmid, we transfected this plasmid and/or microRNA (miRNA)-196a mimic into ESCC cells (Eca109 cell line). Methyl thiazolyl tetrazolium (MTT) assay and Transwell chamber assay were performed to determine cell proliferation, migration and invasion, respectively. Western blot analysis was used to examine the protein expression levels of ANXA1, Snail and E-cadherin. RT-PCR was used to detect the expression of miRNA-196a. Our results revealed that ANXA1 expression was upregulated in the cells transfected with the ANXA1 overexpression plasmid, and cell proliferation, migration and invasion were significantly increased ( $p=0.004, p<0.001$ and $p=0.011$, respectively). In the cells transfected with the miRNA-196a mimic, miRNA-196a expression was significantly upregulated $(\mathrm{p}<0.001)$. However, miRNA-196a expression was downregulated in the cells transfected with the ANXA1 overexpression plasmid. In addition, in the cells transfected with the miRNA-196a mimic, cell proliferation, migration and invasion were significantly decreased $(\mathrm{p}=0.027, \mathrm{p}=0.009$ and $\mathrm{p}=0.021$, respectively). In the cells transfected with the ANXA1 overexpression plasmid, the expression of Snail was upregulated and that of E-cadherin was downregulated. However, the opposite was observed in the cells transfected with the miRNA-196a mimic. Our findings thus demonstrate that ANXA1 promotes the proliferation of Eca109 cells, and increases the expression of Snail, whereas it inhibits that of E-cadherin, thus enhancing the migration and invasion of ESCC cells. miRNA-196a negatively regulates the
\end{abstract}

Correspondence to: Dr Junxing Huang, Department of Oncology, Taizhou People's Hospital Affiliated to Nantong University, 210 Yingchun Road, Taizhou, Jiangsu 225300, P.R. China

E-mail: hhkjdoc@126.com

Key words: Annexin A1, cell proliferation, tumor invasion, esophageal squamous cell carcinoma, microRNA-196a expression of ANXA1, thereby inhibiting the proliferation, invasion and metastasis of ESCC cells.

\section{Introduction}

Esophageal cancer (EC) is a malignant cancer with the $6^{\text {th }}$ highest incidence rate and the $4^{\text {th }}$ highest mortality rate in China (1). The malignancy degree of EC is high, and thus, even following comprehensive treatments, such as surgery, radiotherapy and chemotherapy, the outcomes still remain poor for patients with EC (2). Local recurrence and distant metastasis are the main causes of death in patients with EC, and effective treatments remain a challenge (3). Therefore, investigating the molecular targets and regulatory mechanisms responsible for the proliferation, invasion and metastasis of EC would be of immense practical significance.

Annexin A1 (ANXA1) is an important member of the Annexin superfamily, it can be regulated by $\mathrm{Ca}^{2+}$, it can bind with phospholipids, and participates a variety of physiological-pathological reactions, thus affecting the occurrence, proliferation and apoptosis, and the invasion and metastasis of tumors $(4,5)$; thus, it may be considered as a possible candidate for targeted therapy. It has been indicated that the expression of ANXA1 is associated with the prognosis of esophageal adenocarcinoma (6); however, ANXA1 may exhibit significant differences in expression in different tumors, and opposing results have been obtained (7). Thus, the effects and mechanisms of action of ANXA1 on tumor cells remain unclear. In addition, microRNAs (miRNAs or miRs) can bind with messenger RNAs (mRNAs) of target gene, thus negatively regulating gene expression and affecting the occurrence and progression of a variety of tumors $(8,9)$, Luthra et al reported that miR-196a negatively regulates the expression of the ANXA1 gene, thus affecting the prognosis of esophageal adenocarcinoma (10). In China, the vast majority of EC cases are esophageal squamous cell carcinoma (ESCC), which is significantly different from Western countries, and the expression of ANXA1 differs significantly between esophageal adenocarcinoma and ESCC (11). Therefore, the question of whether the expression of ANXA1 in ESCC affects the proliferation, invasion and metastasis of ESCC cells, as well as the prognosis of ESCC, and whether it is also negatively regulated by miR-196a, is still worthy of investigation. 
In this study, we constructed an ANXA1 overexpression plasmid, and then transfected this plasmid and miR-196a mimics into ESCC Eca109 cells, in an aim to determine whether the overexpression of ANXA1 and miR-196a affects cell proliferation, migration and invasion, and to explore the molecular mechanisms through which miR-196a regulates the expression of ANXA1 and affects the invasion and metastasis of ESCC cells. Our findings may provide the basis for future research on ESCC and may aid in the development of novel treatment strategies for ESCC.

\section{Materials and methods}

Cell and cell culture. The Eca109 cell line was purchased from the Shanghai Institute of Biochemistry and Cell Biology, Chinese Academy of Science (Shanghai, China), and placed in DMEM (Gibco-BRL, Carlsbad, CA, USA) containing 10\% fetal bovine serum (FBS), 2 mmol/1 L-glutamine, $100 \mathrm{U} / \mathrm{ml}$ penicillin and $100 \mu \mathrm{g} / \mathrm{ml}$ streptomycin (Amresco LLC, Solon, $\mathrm{OH}$, USA) at $37^{\circ} \mathrm{C}$ and $5 \%$ of $\mathrm{CO}_{2}$. The medium was changed once every 2 days, and cells in the logarithmic growth phase were used for the experiments.

Construction of ANXA1 overexpression plasmid. TRIzol reagent (Invitrogen Biotechnology Co., Ltd., Shanghai, China) was used to extract RNA from MDA-MB-231 human breast cancer cells (purchased from the Shanghai Institute of Biochemistry and Cell Biology). The AMV reverse transcription kit (Promega, Madison, WI, USA) was then used for reverse transcription to obtain the cDNA which was then used as a template, together with ANXA1 primers (synthesized by Invitrogen Biotechnology Co., Ltd.) for PCR amplification: the primer sequences were as follows: sense, 5'-ATGGCAATGG TATCAGAATTCCTC-3' and antisense, 5'-TTAGTTTCCTC CACAAAGAGCCACC-3'. $\beta$-actin was tested as an internal control, and the primers for $\beta$-actin were as follows: sense primer, GCACCACACCTTCTACAATG and antisense primer, TGCTTGCTGATCCACATCTG (synthesized by Invitrogen Biotechnology Co., Ltd.). PrimeSTAR HS DNA polymerase was purchased from Takara Biotechnology Co.,Ltd. (Dalian, China), and the PCR products were then purified to produce the ANXA1 fragment using the QIAquick PCR Purification kit (Qiagen, Hilden, Germany) following digestion using NdeI and BamHI (both from MBI Fermentas, Burlington, ON, Canada). The pCMV5-myc carrier (Takara Biotechnology Co., Ltd.) was connected internally, via ligase, to generate the expression vector, pCMV5-myc-ANXA1. This expression vector was transfected into DH5 $\alpha$ competent $E$. coli cells following amplification. Subsequently, we used the plasmid DNA kit (purchased from Axygen Biosciences, Union City, CA, USA) to obtain a sufficient amount of expression plasmid, which was subjected to enzyme digestion for identification and sequencing.

Transfection of ANXA1 expression plasmid and miR-196a mimic. The Lipofectamine ${ }^{\mathrm{TM}} 2000 \mathrm{kit}$ (purchased from Invitrogen Biotechnology Co., Ltd.), was used for transfection. Prior to transfection, the ANXA1 overexpression plasmid or miR-196a mimic (designed and synthesized by Shanghai GenePharma Co., Ltd., Shanghai, China) were first mixed with liposomes, allowed to stand at room temperature for $20 \mathrm{~min}$ so as to form a complex, and this complex was then added to the culture wells, following the specific steps included with the kit manual. A nonspecific miRNA mimic (designated as Pre-NC), synthesized by Shanghai GenePharma Co., Ltd., was transfected as an appropriate negative control to miR-196a mimic. The cells transfected with the ANXA1 overexpression plasmid were designated as the ANXA1 group, and those transfected with the miR-196a mimic was designated as the miRNA group; the cells in the empty-vector group were only transfected with empty vectors, and the cells in the control group were untransfected.

Western blot analysis. After the cells were collected, total proteins were extracted using cell lysis, and the DC Protein Assay kit was then used to determine the protein concentrations. A total of $50 \mu \mathrm{g} /$ well protein was then used for gel electrophoresis on $10 \%$ SDS-PAGE gels, and the proteins isolated after electrophoresis were transferred onto polyvinylidene difluoride film (PVDF; Millipore, Billerica, MA, USA). Blocking buffer was then added for $1 \mathrm{~h}$ at room temperature, followed by the addition of the primary antibodies (anti-ANXA1, Cat. no. SAB1405457, 1:1,000; anti-Snail, WH0006591M5, 1:2,000; anti-E-cadherin, WH0000999M1, 1:2,000; and the internal control $\beta$-actin, A1978, 1:3,000) (all from Sigma-Aldrich, St. Louis, MO, USA) for overnight culture at $4{ }^{\circ} \mathrm{C}$ with mild shaking. The membranes were then washed and $2 \%$ BSA-containing horseradish peroxidase-labeled secondary antibody (goat anti-mouse, Cat. no. SAB4600316, Sigma-Aldrich) was then added for $1 \mathrm{~h}$ at room temperature. The membranes were then washed again, and then exposed and developed. Quantity One image analysis software was then used for analysis, and each experiment was repeated 3 times.

Methyl thiazolyl tetrazolium (MTT) assay. The cells from each group were seeded into 96-well plates, with 3 repeated wells for each group of cells. When the cells were in the logarithmic growth phase, MTT solution ( $5 \mathrm{mg} / \mathrm{ml}$, Sigma-Aldrich) was added to each well, followed by culture for a further $4 \mathrm{~h}$ before terminating the culture, and discarding the culture medium. Subsequently, $150 \mu \mathrm{l}$ of dimethyl sulphoxide (DMSO) were added to each well, followed by oscillation in a low-speed shaker for $10 \mathrm{~min}$ to fully dissolve the crystals. The optical density (OD) of each well was then detected using an ELISA detection instrument at $490 \mathrm{~nm}$, and the OD value of each cell was averaged by the 3 repeated wells. The proliferation rate of the cells was then calculated using the following formula: proliferation rate $(\%)=($ mean OD value of the experiment group/mean OD value of the control group) x 100 .

Transwell cell migration/invasion assay. For the migration assay, the cells were collected and resuspended in serum-free DMEM, and then placed in Transwell chambers (Corning Inc., Corning, NY, USA) in 24 well culture plates for $2 \mathrm{~h}$ of culture. FBS-containing complete medium was added to the lower chamber followed by culture for a further $8 \mathrm{~h}$. The cells that had penetrated the polycarbonate membrane of the bottom chamber were then stained with crystal violet (Sigma-Aldrich), and counted under a microscope (Olympus IX53; Olympus Corp., Tokyo, Japan). A total of 10 high-power fields (HPFs) in each chamber were randomly selected for counting, and the mean 
value was set as the number of migrated cells. The procedures for invasion assay were basically the same as those for the migration assay described above, with the exception that a layer of Matrigel was added to the bottom chamber before adding the cells to imitate the extracellular matrix under physiological conditions. Similarly, each chamber was randomly counted at $10 \mathrm{HPFs}$, and the mean value was set as the number of invaded cells.

Reverse transcriptrion-polymerase chain reaction (RT-PCR). Following transfection of the cells with miR-196a mimic for $48 \mathrm{~h}$, and digestion and centrifugation $\left(1,500 \mathrm{xg}, 20^{\circ} \mathrm{C}\right)$, the cells were then collected. TRIzol reagent (Invitrogen Biotechnology Co., Ltd.) was then added to extract the total RNA, and ultraviolet spectrophotometry (Shimadzu UV-2550; Shimadzu Scientific Instruments, Kyoto, Japan)was used to determine the concentration and purity of the RNA. RNA samples with D260 nm/D280 $\mathrm{nm}$ ranging within 1.8 to 2.0 were then selected for reverse transcription and PCR amplification according to the Takara One Step RNA PCR kit (Takara Biotechnology Co., Ltd.) instructions; The RT primer for miR-196a was 5'-GTCAGAAGGAATGATGCACAGCCAAC AACA-3', and the PCR primers were 5'-CGTCAGAAGGAA TGATGCACAG-3' (forward) and 5'-ACCTGCGTAGGTA GTTTCATGT-3' (reverse). U6 was used as an internal reference and the U6 primer for RT was 5'-AACGCTTCACGAA TTTGCGT-3', and the PCR primers were 5'-CTCGCTTCGGC AGCACA3' (forward) and 5'-AACGCTTCACGAATTT GCGT-3' (reverse). All those primers for miR-196a and U6 were synthesized by Invitrogen Biotechnology Co., Ltd. The PCR reaction conditions were as follows: pre-denaturation at $95^{\circ} \mathrm{C}$, $15 \mathrm{~min}$; denaturation at $95^{\circ} \mathrm{C}, 15 \mathrm{sec}$; annealing and extension at $60^{\circ} \mathrm{C}, 1 \mathrm{~min}, 40$ cycles. The $2^{-\Delta \Delta \mathrm{CT}}$ value (in which $\left.\Delta \mathrm{CT}=\mathrm{CT}_{\text {sample }}-\mathrm{CT}_{\text {internal reference }}\right)$ was used to represent the relative expression level of the target miRNA. The experiment was repeated 3 times for the average.

Statistical analysis. The experimental data are expressed as the average of triplicate experiments and are the means \pm standard deviation. Comparisons of the measurement data were made using the two-tailed Student's t-test, and comparisons of the migration rates and invasion rates of the cells in the different groups were made by analysis of variance. A value of $p<0.05$ was considered to indicate a statistically significant difference. The SPSS 19.0 software package (SPSS, Inc., Chicago, IL, USA) was used for statistical analysis.

\section{Results}

Successful transfection of ANXA1 plasmid into Ecal09 cells. After the ANXA1 overexpression plasmid was transfected into the Eca109 cells, western blot analysis was used to measure the protein expression levels. The results revealed that the cells in the ANXA1 group exhibited an obvious ANXA1 band (Fig. 1), indicative of a significantly higher protein expression compared with the cells in the empty-vector group. This indicated that transfection with the ANXA1 overexpression plasmid efficiently upregulated the expression of ANXA1.

Effect of the overexpression of ANXA1 on the proliferation of Ecal09 cells. MTT assay at 24, 48 and 72 h post-transfection

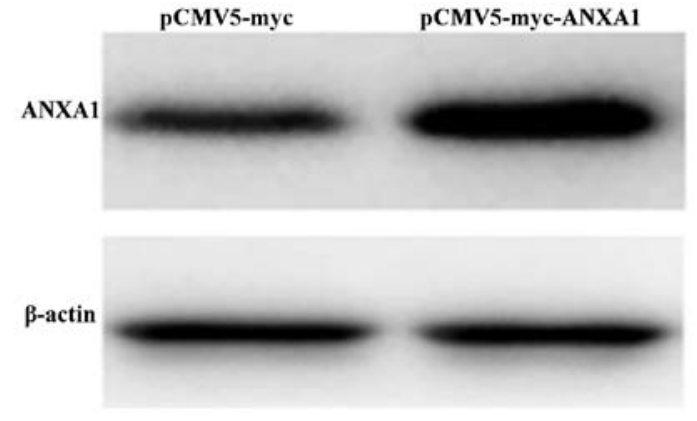

Figure 1. Western blot analysis of Annexin A1 (ANXA1) expression. ANXA1 expression was upregulated in the cells transfected with the ANXA1 expression plasmid, and the band of the ANXA1in the ANXA1 group was significantly thicker than that of the empty vector group.

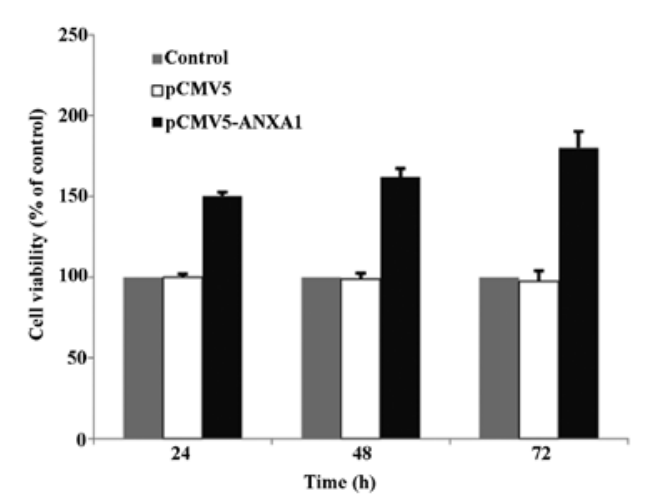

Figure 2. MTT assay showing the relative survival rates of the cells in the different groups. At 24, 48 and $72 \mathrm{~h}$ post-transfection, the relative survival rate in the Annexin A1 (ANXA1) group was significantly higher than that of the empty-vector group at 24,48 and $72 \mathrm{~h}$ post-transfection $(\mathrm{p}=0.004, \mathrm{p}<0.001$ and $\mathrm{p}<0.001$, respectively; asterisks indicate significant differences compared to the emtpy-vector group). The survival rates of the cells in the empty-vector group and the control group were similar, close to $100 \%$.

revealed that the survival rate of the cells in the empty-vector group was similar to that of the cells in the control group, indicating that the transfection of the vector did not affect cell proliferation. However, the survival rate of the cells in the ANXA1 group was significantly increased at 24-72 h posttransfection (150-180\%), suggesting that ANXA1 promotes cell proliferation; this promoting effect on proliferation was more pronounced as time progressed (Fig. 2).

Effect of the overexpression of ANXAI on the migration and invasion of Ecal09 cells. The results of Transwell migration assay revealed that a significantly greater number of cells in the ANXA1 group had migrated to the bottom surface of the chamber compared to the cells in the empty-vector group (240 cells/HPF vs. 123 cells/HPF, p<0.001; Fig. 3A). Similarly, the results of invasion assay revealed that the number of cells in the ANXA1 group that penetrated the basement membrane was significantly increased than those in the empty-vector group (79 cells/HPF vs. 36 cells/HPF, $\mathrm{p}=0.011$; Fig. $3 \mathrm{~B}$ ), indicating that the overexpression of ANXA1 enhanced the cell migration and invasion ability.

Effect of the overexpression of miRNA-196a on the expression of ANXA1. The results of RT-PCR indicated that following transfection of the cells with miR-196a mimic, the expression 
A

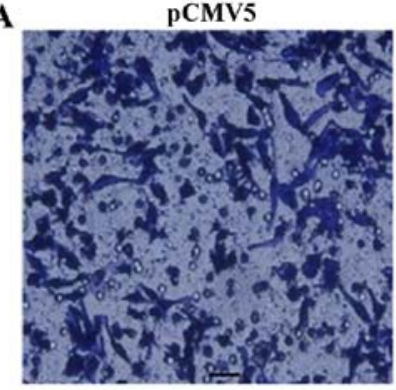

pCMV5

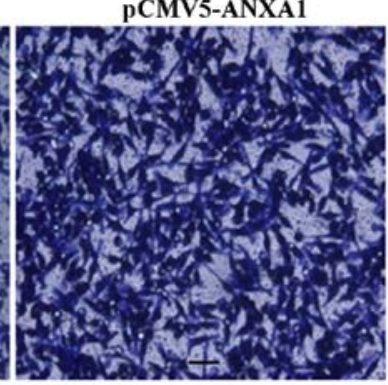

B

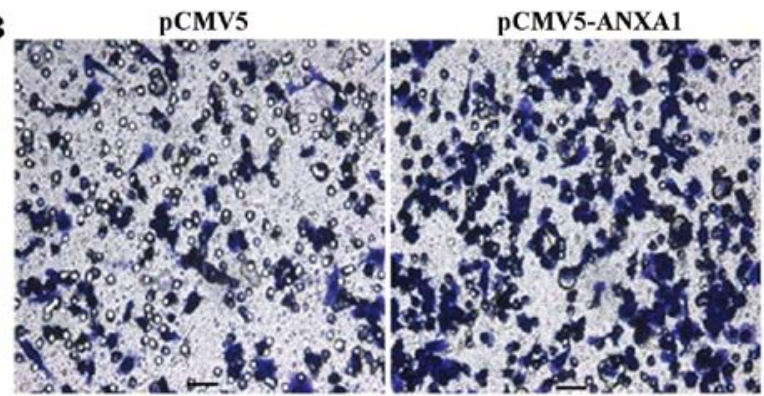

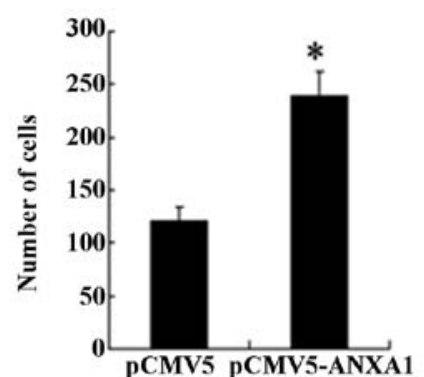

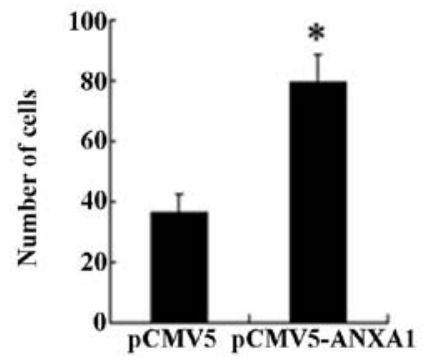

Figure 3. Transwell migration and invasion assay. (A and B) Left panels show microscopic images of cells in the Annexin A1 (ANXA1) group and empty-vector group (crystal violet staining, x200 magnification). The ANXA1 group exhibited a greater number of cells entering the bottom of the chamber. Right panels are comparisons of cell counting in the ANXA1 and empty-vector groups. The ANXA1 group exhibited a greater number of invading and migrating cells than in the empty-vector group $(\mathrm{p}<0.001$ and $\mathrm{p}=0.011$; asterisks indicate significant differences).

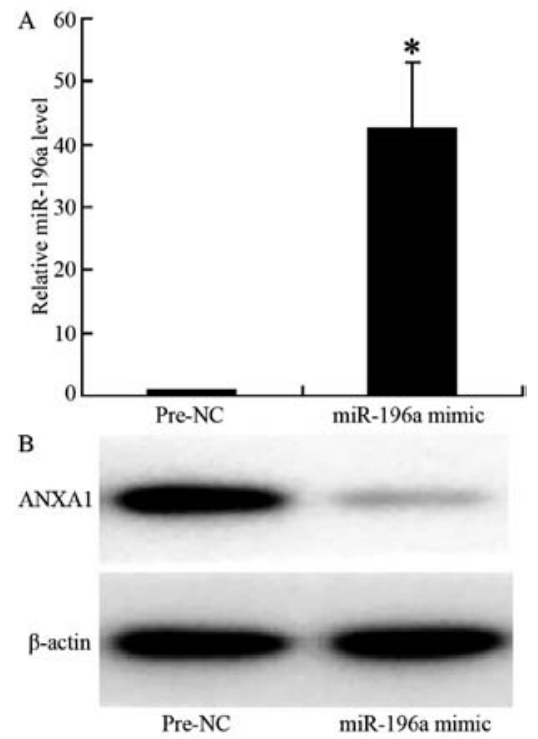

Figure 4. Expression of miR-196a and Annexin A1 (ANXA1) following the transfection of miR-196a mimic into the Eca109 cells. (A) The expression of miR-196a was significantly higher in the miR-196a mimic-transfected cells compared to the control group (Pre-NC; ${ }^{*} \mathrm{p}<0.001$ ). (B) ANXA1 expression was downregulated in the cells transfected with the miR-196a mimic, and the band became significantly lighter.

of miR-196a in the cells was significantly increased (relative expression level, 1.1 vs. 43, p $<0.001$; Fig. 4A). The results of western blot analysis revealed that ANXA1 protein expression was significantly downregulated in the cells transfected with miR-196a mimic, with a significantly lighter electrophoretic band (Fig. 4B), indicating that the overexpression of miR-196a significantly decreased the expression of ANXA1, thus confirming that miR-196a negatively regulates the expression of ANXA1 in ESCC cells.
Effect of miR-196a overexpression on the proliferation, migration and invasion of Ecal09 cells. The results of MTT assay revealed that the survival rate of the cells transfected with the miR-196a mimic was significantly lower than that of the cells in the control group (Pre-NC group; 57 vs. 100\%, $\mathrm{p}=0.027$ ) (Fig. 5A). However, co-transfection of the cells with the miR-196a mimic and the ANXA1 overexpression plasmid reversed the inhibitory effects of miR-196a on cell proliferation (90 vs. 57\%, p=0.034) (Fig. 5A). The results of Transwell chamber assay revealed that cell migration and invasion were significantly decreased when miR-196a was overexpressed in the cells (56 vs. $116, \mathrm{p}=0.009 ; 24$ vs. $46, \mathrm{p}=0.021$, respectively) (Fig. 5B and $\mathrm{C}$ ). However, co-transfection of the cells with miR-196a mimic and the ANXA1 overexpression plasmid reversed the inhibitory effects of miR-196a on cell migration and invasion ( 99 vs. $56, \mathrm{p}=0.015 ; 38$ vs. $24, \mathrm{p}=0.04$, respectively) (Fig. 5B and $\mathrm{C}$ ). These results confirmed that miR-196a decreases ANXA1 expression, thereby inhibiting the proliferation, migration and invasion of Eca109 cells.

Association between the expression of ANXA1, Snail and $E$-cadherin. Our results revealed that following the overexpression of ANXA1, the expression of E-cadherin in the Eca109 cells was decreased, while that of the transcription factor Snail was upregulated (Fig. 6A). However, following the overexpression of miR-196a, the expression of E-cadherin was upregulated, while that of Snail was downregulated (Fig. 6B).

\section{Discussion}

ANXA1 (also known as lipocortin 1, phospholipase A2 inhibitory protein) is a member of the Annexin family, and belongs to calcium-dependent phospholipid binding protein. It was previously found that ANXA1 was an intracellular inflamma- 
A
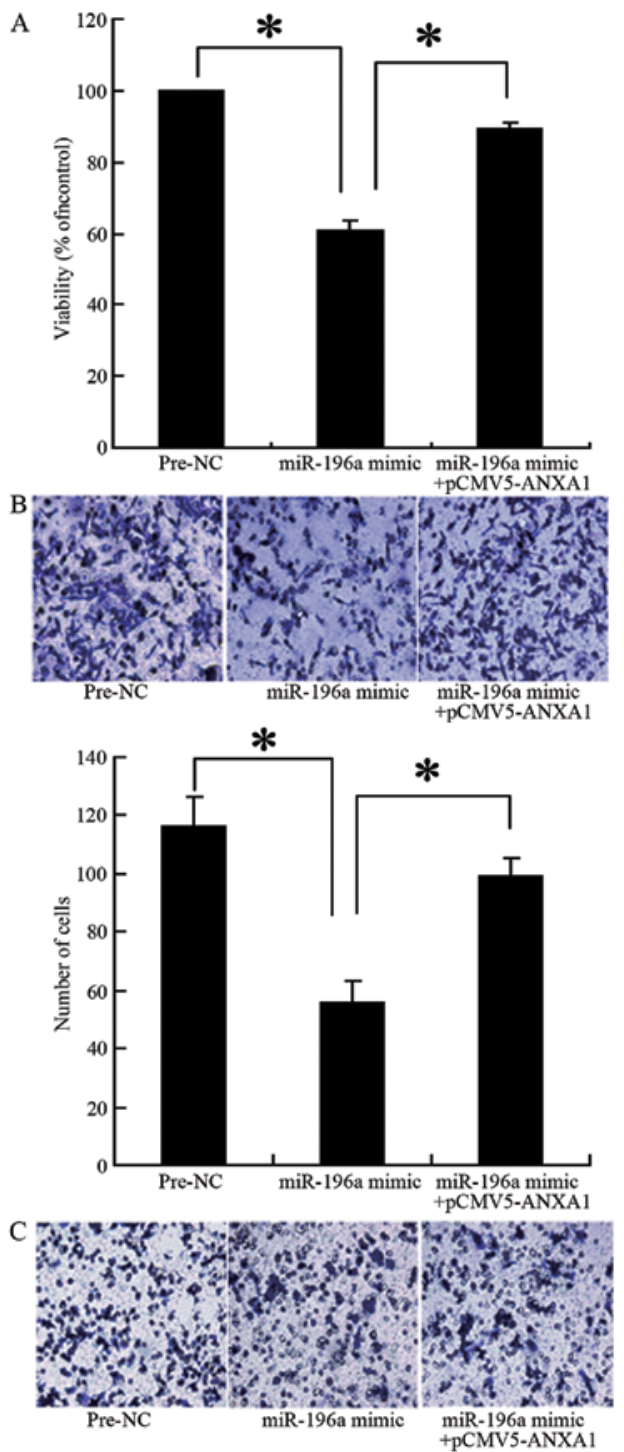

$*$

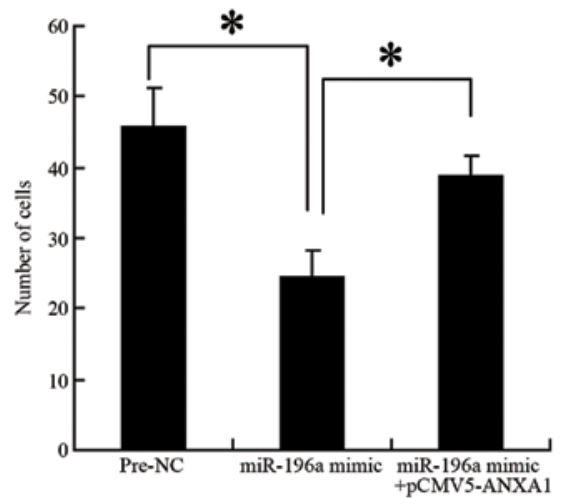

Figure 5. Effect of miR-196a upregulation and Annexin A1 (ANXA1) overexpression on cell proliferation, migration and invasion. (A) The overexpression of miR-196a inhibited the expression of ANXA1, thereby inhibiting cell proliferation $(\mathrm{p}=0.027)$; co-transfection with ANXA1 expression plasmid reversed this inhibitory effect $(\mathrm{p}=0.034)$; (B) the overexpression of miR-196a inhibited cell migration $(\mathrm{p}=0.009)$; co-transfection with ANXA1 expression plasmid promoted cell migration $(\mathrm{p}=0.015)$; (C) the overexpression of miRNA-196a inhibited cell invasion ( $\mathrm{p}=0.021)$; co-transfection with ANXA1 expression plasmid promoted cell invasion $(\mathrm{p}=0.045)$. Asterisks indicate significant differences with the indicated $\mathrm{p}$-values.

tion-related factor that regulates the anti-inflammatory effects of glucocorticoids, and it has also been shown to be involved in the

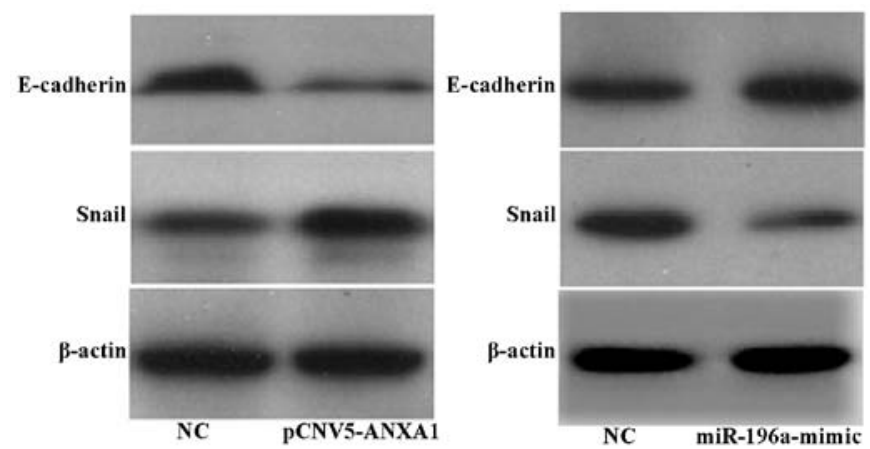

Figure 6. (A) The overexpression of Annexin A1 (ANXA1) upregulates Snail and downregulates E-cadherin expression in Eca109 cells; (B) miR-196a mimic inhibited the expression of ANXA1, followed by the downregulated expression of Snail and the upregulated expression of E-cadherin in Eca109 cells (with $\beta$-actin as the internal control).

regulation of cell proliferation, differentiation and apoptosis, as well as endocytosis and secretion, and to be closely related to the occurrence or progression of tumors (12 and refs. therein). Certain conflicting results have been found in the published studies on the association between ANXA1 and malignant tumors: firstly, ANXA1 is differently expressed in different malignancies; in gastric cancer, pancreatic cancer, liver cancer, esophageal adenocarcinoma and glioma, ANXA1 has been shown to be upregulated (13-16); however, it has been found to be downregulated or even absent in prostate cancer, ESCC, breast cancer and B-cell lymphoma (17-20). Secondly, there are differences in the understanding of the roles of ANXA1 in tumorigenesis. In some tumors, ANXA1 has been shown to stimulate cell proliferation and promote the invasion and metastasis of tumors (14,21-24), whereas in other tumors, ANXA1 has been shown to exhibit the characteristics of a tumor suppressor gene, such as inhibiting cell proliferation and inducing apoptosis $(11,25-28)$. Thus, the expression of ANXA1 in malignant tumors seems to be tumor-specific, and plays multi-factorial roles.

Our results confirmed that ANXA1 promoted the proliferation of ESCC cells; however, but the exact mechanisms involved remain unclear. The possible mechanisms responsible for the promoting effects of ANXA1 on cell proliferation may include: i) ANXA1 has multiple phosphorylation sites, and can act as the receptor substrate of EGF/HGF and other growth signals, when proliferated by protein kinase $\mathrm{C}$ (PKC) or tyrosine kinase (TK), and it may then activate IKK, NF- $\kappa \mathrm{B}$ and other downstream molecules, thus promoting cell proliferation (29-31); ii) combined with formyl peptide receptors (FPRs) on the cell surface, it participates in the induction of mitogen-activated proliferation signals $(32,33)$; iii) it acts as the transcriptional target of forkhead transcription factor FoxM1, thus promoting the proliferation, invasion, metastasis and angiogenesis of tumor cells (34) and iv) it inhibits tumor necrosis factor-induced apoptosis (35). However, there is evidence to indicate that ANXA1 can inhibit cell proliferation, as demonstrated in the study by Alldridge and Bryant, who reported that ANXA1 activated the ERK1/2 and MAPK signal transduction pathways, thus destroying the cytoskeleton, inhibiting the expression of cyclin D1 and inhibiting cell proliferation (36). By inhibiting COX-2, ANXA1 has been shown to inhibit the proliferation of gastric cancer cells (11). It is unclear as to why ANXA1 can 
promote cell proliferation in some tumors, while it inhibits it in others; this may be due to the different phenotypes of ANXA1. $\mathrm{Hu}$ et al analyzed the mutations in the promoter region and the coding region of the whole ANXA1 gene, and did not find any mutation or polymorphism (37) so as to support this hypothesis. Thus, further studies are warranted to elucidate the mechanisms through which ANXA1 affects the proliferation of ESCC cells.

This study also found that the overexpression of ANXA1 promoted the migration and invasion of ESCC Eca109 cells; the enhanced cell migration, invasion and growth are closely related to clinical metastasis and progression. Thus, this study suggested that ANXA1 promotes the progression and metastasis of ESCC, consistent with other studies in which ANXA1 has been reported to be able to promote the invasion and metastasis of gastric cancer, pancreatic cancer, breast cancer, lung cancer and colorectal cancer (14,38-42). However, other studies have found opposite results, demonstrating that ANXA1 inhibits the growth, invasion and metastasis of nasopharyngeal carcinoma (43), and that the knockdown of ANXA1 promoted cell proliferation and invasion (44).

Tumor invasion and metastasis is a complex process, with tumor cells shedding from the primary sites as the first step, which is related to the reduced intercellular adhesion functions and epithelial-mesenchymal transition(EMT).EMT refers to the process that polar, mutually adhered epithelial cells transform into stromal cell-like substances that are active and can move freely within the intercellular matrix. EMT occurs in the early stage of the cascade of tumor invasion and metastasis, during which the downregulation of the adhesion-associated protein, E-cadherin, is the most important molecular event $(45,46)$. E-cadherin is a member of the cadherin family, and mediates the adhesion of cells, thus playing important roles in maintaining cell polarity and the structural integrity of the tissues. The transcription factor Snail binds with E-box in the promoter region of E-cadherin, thus downregulating E-cadherin and promoting cell shedding and migration (47-49); thus, it is closely related to the in situ invasion and remote metastasis of tumor cells. The results of this study demonstrated that the overexpression of ANXA1 upregulated Snail expression, while it downregulated that of E-cadherin. However, when the expression of ANXA1 was decreased by transfection with miR-196a mimic, Snail expression was then downregulated, while E-cadherin expression was upregulated, confirming that the ANXA1 gene exerts its effects on the invasion and metastasis of ESCC cells through the Snail/E-cadherin pathway.

It had been reported that the 3 '-UTR sequences of the ANXA1 gene mRNA and 5'-end sequence of miR-196a are highly consistent, and ANXA1 is the target gene of miR-196a. When ANXA1 mRNA and miR-196a are combined with each other, this can lead to the formation of a gene-silencing complex, thus blocking the protein translation of ANXA1 $(10,50)$. This study found that miR-196a downregulated the expression of ANXA1, thus inhibiting the expression of Snail, while promoting the expression of E-cadherin, as well as inhibiting the proliferation, invasion and metastasis of ESCC cells, consistent with the findings of other studies $(51,52)$. Based on the oncogeneic effects of ANXA1 (promoting the proliferation, invasion and metastasis of ESCC cells), it may be conceivable to use miR-196a in the treatment of patients with ESCC who exhibit a high ANXA1 expression, as well as with a high risk of metastasis/recurrence.
As miR-196a is only approximately 21 bases, it can be stably expressed long-term in vivo, and it would not interfere with host DNA replication and transcription; thus, it would not lead to iatrogenic mutation. The use of miR-196a may thus be a promising new approach for the treatment of ESCC.

In conclusion, we demonstrate that the overexpression of ANXA1 promotes the proliferation of ESCC cells, and promotes the invasion and metastasis of ESCC cells by increasing the expression of the transcription factor Snail, while inhibiting that of E-cadherin. miR-196a may downregulate ANXA1, thus negatively regulating ANXA1 and inhibiting the Snail/E-cadherin pathway, followed by the inhibition of EMT, and ultimately the inhibition of the proliferation, invasion and metastasis of ESCC cells. The results of this study may be used to screen patients with EC who are at risk of recurrence/metastasis. Our findings may prove useful in the development of drugs targeting ANXA1 and miR-196a, thus providing new treatment methods, apart from conventional radiotherapy, for the treatment of high-risk patients with ESCC who exhibit a high expression of ANXA1.

\section{Acknowledgements}

This study was supported by the Jiangsu Province Ministry of Health, China (grant.no. H201260) and the Taizhou Committee of Science and Technology, China (grant. no. TS201346).

\section{References}

1. Chen W, Zheng R, Zeng H, Zhang S and He J: Annual report on status of cancer in China, 2011. Chin J Cancer Res 27: 2-12, 2015.

2. Yan W, Wistuba II, Emmert-Buck MR and Erickson HS: Squamous cell carcinoma - similarities and differences among anatomical sites. Am J Cancer Res 1: 275-300, 2011.

3. Matsuda S, Takeuchi H, Kawakubo H, Ando N and Kitagawa Y: Current advancement in multidisciplinary treatment for resectable cStage II/III esophageal squamous cell carcinoma in Japan. Ann Thorac Cardiovasc Surg. 22: 275-283, 2016.

4. Lim LH and Pervaiz S: Annexin 1: the new face of an old molecule. FASEB J 21: 968-975, 2007.

5. Guo C, Liu S and Sun MZ: Potential role of Anxal in cancer. Future Oncol 9: 1773-1793, 2013.

6. Wang KL, Wu TT, Resetkova E, Wang H, Correa AM, Hofstetter WL, Swisher SG, Ajani JA, Rashid A, Hamilton SR and Albarracin C: Expression of Annexin A1 in esophageal and esophagogastric junction adenocarcinomas: association with poor outcome. Clin Cancer Res 12: 4598-4604, 2006.

7. Bhardwaj A, Ganesan N, Tachibana K, Rajapakshe K, Albarracin CT, Gunaratne PH, Coarfa C and Bedrosian I: Annexin A1 preferentially predicts poor prognosis of basal-like breast cancer patients by activating mTOR-S6 signaling. PLoS One 10: e0127678, 2015.

8. Zhang AX, Lu FQ, Yang YP, Ren XY, Li ZF and Zhang W: MicroRNA-217 overexpression induces drug resistance and invasion of breast cancer cells by targeting PTEN signaling. Cell Biol Int: Jun 24, 2015 (Epub ahead of print).

9. Lynam-Lennon N, Reynolds JV, Marignol L, Sheils OM, Pidgeon GP and Maher SG: MicroRNA-31 modulates tumour sensitivity to radiation in oesophageal adenocarcinoma. J Mol Med (Berl) 90: 1449-1458, 2012.

10. Luthra R, Singh RR, Luthra MG, Li YX, Hannah C, Romans AM, Barkoh BA, Chen SS, Ensor J, Maru DM, et al: MicroRNA-196a targets Annexin A1: a microRNA-mediated mechanism of Annexin A1 downregulation in cancers. Oncogene 27: 6667-6678, 2008.

11. Gao Y, Chen Y, Xu D, Wang J and Yu G: Differential expression of ANXA1 in benign human gastrointestinal tissues and cancers. BMC Cancer 14: 520, 2014.

12. Mussunoor S and Murray GI: The role of Annexins in tumour development and progression. J Pathol 216: 131-140, 2008. 
13. Bai XF, Ni XG, Zhao P, Liu SM, Wang HX, Guo B, Zhou LP, Liu F, Zhang JS, Wang K, et al: Overexpression of Annexin 1 in pancreatic cancer and its clinical significance. World J Gastroenterol 10: 1466-1470, 2004.

14. Lin Y, Lin G, Fang W, Zhu H and Chu K: Increased expression of Annexin A1 predicts poor prognosis in human hepatocellular carcinoma and enhances cell malignant phenotype. Med Oncol 31: 327, 2014.

15. Xue LY, Teng LH, Zou SM, Ren LQ, Zheng S, Luo W, Bi R and Lü N: Expression of Annexin I in different histological types of carcinomas. Zhonghua Zhong Liu Za Zhi 29: 444-448, 2007.

16. Zhang ZQ, Li XJ, Liu GT, Xia Y, Zhang XY and Wen H: Identification of Annexin A1 protein expression in human gastric adenocarcinoma using proteomics and tissue microarray. World J Gastroenterol 19: 7795-7803, 2013.

17. Kang JS, Calvo BF, Maygarden SJ, Caskey LS, Mohler JL and Ornstein DK: Dysregulation of Annexin I protein expression in high-grade prostatic intraepithelial neoplasia and prostate cancer. Clin Cancer Res 8: 117-123, 2002.

18. Shen D, Nooraie F, Elshimali Y, Lonsberry V, He J, Bose S, Chia D, Seligson D, Chang HR and Goodglick L: Decreased expression of Annexin A1 is correlated with breast cancer development and progression as determined by a tissue microarray analysis. Hum Pathol 37: 1583-1591, 2006.

19. Vishwanatha JK, Salazar E and Gopalakrishnan VK: Absence of Annexin I expression in B-cell non-Hodgkin's lymphomas and cell lines. BMC Cancer 4: 8, 2004.

20. Xia SH, Hu H, Hu LP, Xu X, Cai Y, Han YL, Chen BS, Wei F Ying WT, Qian XH, et al: Analysis of proteins with altered expression in human esophageal squamous cell carcinomas. A Zheng 21: 11-15, 2002 (In Chinese).

21. Sato Y, Kumamoto K, Saito K, Okayama H, Hayase S, Kofunato Y, Miyamoto K, Nakamura I, Ohki S, Koyama Y, et al: Up-regulated Annexin A1 expression in gastrointestinal cancer is associated with cancer invasion and lymph node metastasis. Exp Ther Med 2: 239-243, 2011.

22. Woś M and Bandorowicz-Pikuła J: Participation of Annexins in endocytosis and EGFR-mediated signal transduction. Postepy Biochem 60: 55-61, 2014 (In Polish).

23. Sheu MJ, Li CF, Lin CY, Lee SW, Lin LC, Chen TJ and Ma LJ: Overexpression of ANXA1 confers independent negative prognostic impact in rectal cancers receiving concurrent chemoradiotherapy. Tumour Biol 35: 7755-7763, 2014.

24. Yi M and Schnitzer JE: Impaired tumor growth, metastasis, angiogenesis and wound healing in Annexin A1-null mice. Proc Natl Acad Sci USA 106: 17886-17891, 2009.

25. Hsiang $\mathrm{CH}$, Tunoda T, Whang YE, Tyson DR and Ornstein DK The impact of altered Annexin I protein levels on apoptosis and signal transduction pathways in prostate cancer cells. Prostate 66 $1413-1424,2006$

26. Wang LP, Bi J, Yao C, Xu XD, Li XX, Wang SM, Li ZL, Zhang DY, Wang $M$ and Chang GQ: Annexin A1 expression and its prognostic significance in human breast cancer. Neoplasma 57 253-259, 2010

27. Ang EZ, Nguyen HT, Sim HL, Putti TC and Lim LH: Annexin-1 regulates growth arrest induced by high levels of estrogen in MCF-7 breast cancer cells. Mol Cancer Res 7: 266-274, 2009.

28. Mu D, Gao Z, Guo H, Zhou G and Sun B: Sodium butyrate induces growth inhibition and apoptosis in human prostate cancer DU145 cells by up-regulation of the expression of Annexin A1. PLoS One 8: e74922, 2013.

29. D'Acunto CW, Gbelcova H, Festa M and Ruml T: The complex understanding of Annexin A1 phosphorylation. Cell Signal 26 173-178, 2014.

30. Bist P, Leow SC, Phua QH, Shu S, Zhuang Q, Loh WT, Nguyen TH, Zhou JB, Hooi SC and Lim LH: Annexin-1 interacts with NEMO and RIP1 to constitutively activate IKK complex and $\mathrm{NF}-\kappa \mathrm{B}$ : implication in breast cancer metastasis. Oncogene 30 3174-3185, 2011

31. Hoque M, Rentero C, Cairns R, Tebar F, Enrich C and Grewal T: Annexins - scaffolds modulating PKC localization and signaling. Cell Signal 26: 1213-1225, 2014.

32. Khau T, Langenbach SY, Schuliga M, Harris T, Johnstone CN, Anderson RL and Stewart AG: Annexin-1 signals mitogen-stimulated breast tumor cell proliferation by activation of the formyl peptide receptors (FPRs) 1 and 2. FASEB J 25: 483-496, 2011.

33. Gastardelo TS, Cunha BR, Raposo LS, Maniglia JV, Cury PM, Lisoni FC, Tajara EH and Oliani SM: Inflammation and cancer: role of Annexin A1 and FPR2/ALX in proliferation and metastasis in human laryngeal squamous cell carcinoma. PLoS One 9: e111317, 2014
34. Cheng SX, Tu Y and Zhang S: FoxM1 promotes glioma cells progression by up-regulating Anxa1 expression. PLoS One 8: e72376, 2013.

35. Wu YL, Jiang XR, Lillington DM, Newland AC and Kelsey SM: Upregulation of lipocortin 1 inhibits tumour necrosis factor-induced apoptosis in human leukaemic cells: a possible mechanism of resistance to immune surveillance. Br J Haematol 111: 807-816, 2000

36. Alldridge LC and Bryant CE: Annexin 1 regulates cell proliferation by disruption of cell morphology and inhibition of cyclin D1 expression through sustained activation of the ERK1/2 MAPK signal. Exp Cell Res 290: 93-107, 2003.

37. Hu N, Flaig MJ, Su H, Shou JZ, Roth MJ, Li WJ, Wang C, Goldstein AM, Li G, Emmert-Buck MR and Taylor PR: Comprehensive characterization of Annexin I alterations in esophageal squamous cell carcinoma. Clin Cancer Res 10: 60136022,2004

38. Okano M, Kumamoto K, Saito M, Onozawa H, Saito K, Abe N, Ohtake T and Takenoshita S: Upregulated Annexin A1 promotes cellular invasion in triple-negative breast cancer. Oncol Rep 33: 1064-1070, 2015

39. Babbin BA, Lee WY, Parkos CA, Winfree LM, Akyildiz A Perretti $\mathrm{M}$ and Nusrat A: Annexin I regulates SKCO-15 cell invasion by signaling through formyl peptide receptors. J Biol Chem 281: 19588-19599, 2006.

40. Kim SW, Rhee HJ, Ko J, Kim YJ, Kim HG, Yang JM, Choi EC and Na DS: Inhibition of cytosolic phospholipase A2 by Annexin I. Specific interaction model and mapping of the interaction site. J Biol Chem 276: 15712-15719, 2001.

41. Belvedere R, Bizzarro V, Popolo A, Dal Piaz F, Vasaturo M, Picardi P, Parente L and Petrella A: Role of intracellular and extracellular Annexin A1 in migration and invasion of human pancreatic carcinoma cells. BMC Cancer 14: 961, 2014.

42. Cheng TY, Wu MS, Lin JT, Lin MT, Shun CT, Huang HY, Hua KT and Kuo ML: Annexin A1 is associated with gastric cancer survival and promotes gastric cancer cell invasiveness through the formyl peptide receptor/extracellular signal-regulated kinase/integrin beta-1-binding protein 1 pathway. Cancer 118: 5757-5767, 2012

43. Liu A, Huang W, Zeng G, Ma X, Zhou X, Wang Y, Ouyang C and Cheng A: Expression of the Annexin A1 gene is associated with suppression of growth, invasion and metastasis of nasopharyngeal carcinoma. Mol Med Rep 10: 3059-3067, 2014.

44. Suh YE, Raulf N, Gäken J, Lawler K, Urbano TG, Bullenkamp J, Gobeil S, Huot J, Odell E and Tavassoli M: MicroRNA-196a promotes an oncogenic effect in head and neck cancer cells by suppressing Annexin A1 and enhancing radioresistance. Int J Cancer 137: 1021-1034, 2015.

45. Buda A and Pignatelli M: E-cadherin and the cytoskeletal network in colorectal cancer development and metastasis. Cell Commun Adhes 18: 133-143, 2011.

46. Gheldof A and Berx G: Cadherins and epithelial-to-mesenchymal transition. Prog Mol Biol Transl Sci 116: 317-336, 2013.

47. Peinado H, Ballestar E, Esteller M and Cano A: Snail mediates E-cadherin repression by the recruitment of the Sin3A/histone deacetylase 1 (HDAC1)/HDAC2 complex. Mol Cell Biol 24: 306-319, 2004

48. Wang YL, Zhao XM, Shuai ZF, Li CY, Bai QY, Yu XW and Wen QT: Snail promotes epithelial-mesenchymal transition and invasiveness in human ovarian cancer cells. Int J Clin Exp Med 8: 7388-7393, 2015

49. Montserrat N, Gallardo A, Escuin D, Catasus L, Prat J, Gutiérrez-Avignó FJ, Peiró G, Barnadas A and Lerma E: Repression of E-cadherin by SNAIL, ZEB1, and TWIST in invasive ductal carcinomas of the breast: a cooperative effort? Hum Pathol 42: 103-110, 2011.

50. Engels BM and Hutvagner G: Principles and effects of microRNA-mediated post-transcriptional gene regulation. Oncogene 25: 6163-6169, 2006.

51. Pin AL, Houle F, Fournier P, Guillonneau M, Paquet ÉR, Simard MJ, Royal I and Huot J: Annexin-1-mediated endothelial cell migration and angiogenesis are regulated by vascular endothelial growth factor (VEGF)-induced inhibition of miR-196a expression. J Biol Chem 287: 30541-30551, 2012.

52. Swa HL, Blackstock WP, Lim LH and Gunaratne J: Quantitative proteomics profiling of murine mammary gland cells unravels impact of Annexin-1 on DNA damage response, cell adhesion, and migration. Mol Cell Proteomics 11: 381-393, 2012. 